\title{
A Longitudinal Study of The Nasal Symmetry in Unilateral Cleft Lip And Palate Patients Treated With Nasoalveolar Molding
}

\author{
Tulasi Nayak ${ }^{1}$ Renu Parmar ${ }^{2} \quad$ Krishnamurthy Bonanthaya ${ }^{2}$ Pritham Shetty ${ }^{2}$ \\ ${ }^{1}$ Department of Oral and Maxillofacial Surgery, Dayananda Sagar \\ College of Dental Sciences, Jayanagar, Bengaluru, India \\ 2Department of Oral and Maxillofacial Surgery, Smile Train Cleft \\ Centre, Bhagwan Mahaveer Jain Hospital, Bangalore

\begin{abstract}
Address for correspondence Nayak Tulasi, BDS MDS, Department of Oral and Maxillofacial Surgery, Dayananda Sagar College of Dental Sciences, Jayanagar, 4th T Block, Bengaluru, 560041, India (e-mail: drtulasinayak@gmail.com).
\end{abstract}

\begin{abstract}
Keywords

- cleft lip

- cleft nose

- long term

- nasal symmetry

- nasoalveolar molding

Background Nasoalveolar molding (NAM) has been extensively used as a presurgical technique in the treatment of unilateral cleft lip and palate (UCLP) over the last two decades. It has proven to be a useful tool to reduce the cleft size, improve nasal symmetry, and increase the columellar length. The long-term stability of these findings has not been conclusively proven.

Methods In this longitudinal study, the nasal symmetry of 24 NAM treated UCLP patients was evaluated to assess the 5-year stability of NAM. The basal photographs were shot postcheiloplasty (T1), at 1-year follow-up (T2), at 3-year follow-up (T3), and 5-year follow-up (T4) appointments.

Results In this study, we found that NAM was a useful adjuvant in achieving nasal symmetry in patients with UCLP in the immediate postoperative period. However, as the patients aged, there was a gradual loss of mean nasal height (by 22.83\%) and columella length (by $24.89 \%$ ), a mean gain in nasal width (by $40.25 \%$ ) and alar base width (by $40.69 \%$ ), and an increase in the columella deviation (by 3.46\%) from the T1 to the T4 follow-up.

Conclusion Although there is no conclusive evidence, the loss of symmetry may be due to the unequal growth on the cleft and noncleft sides. These patients will be followed-up till end of growth for a definite conclusion on the long-term effect of NAM.
\end{abstract}

\section{Introduction}

The unilateral cleft nasal deformity is a common technical challenge faced by surgeons around the world. Although multiple techniques ${ }^{1-5}$ have been used with varying success rates to improve symmetry, absolute long-term symmetry still remains an elusive goal. The presurgical nasoalveolar molding (NAM) technique proposed by Grayson ${ }^{6}$ has been gaining popularity over the last two decades due to its utility in reducing the severity of the of clefts in early infancy. ${ }^{7}$ Although multiple short-term studies, ${ }^{8-12}$ have shown an increase in the columella length, molding of the deformed nasal cartilages, and obtaining arch approximation, the

published online

August 4, 2020
DOI https://doi.org/

10.1055/s-0040-1714768 ISSN 0970-0358. retention of the results obtained have not been conclusively established. ${ }^{1}$ We have conducted this longitudinal study to ascertain the 5-year stability of the nasal symmetry achieved by NAM in unilateral cleft lip and palate (UCLP) patients.

\section{Methodology}

This research was conducted in accordance with the Helsinki Declaration. In this retrospective study, consecutive NAM-treated UCLP patients from 2012-2013, whose serial photographs were available, were studied. Patients with incomplete clefts and other craniofacial anomalies were 
excluded from this study. All infants who had arrived at the center before 6 weeks of age were treated with Eric Liou's technique of $\mathrm{NAM}^{13}$ for an average of 4 months. The patients were called every 2 weeks to make adjustments to the device. Six weeks was used as a cutoff, since the pliability of the nasal cartilage reduces as the child ages (-Figs. 1 and 2). ${ }^{14}$ The patients were treated with modified Millard's cheiloplasty at an average age of 6 months of age without primary rhinoplasty or gingivoperiosteoplasty. One-stage palatoplasty was carried out along with Bardach's technique, with repositioning of the soft palate musculature (Sommerlad). ${ }^{15}$ All the surgeries were performed by the same two surgeons. A total of 24 patients were included in this study. Standardized 2D full-face frontal view and worm's eye photographs were shot by the same experienced cleft worker postcheiloplasty (T1),

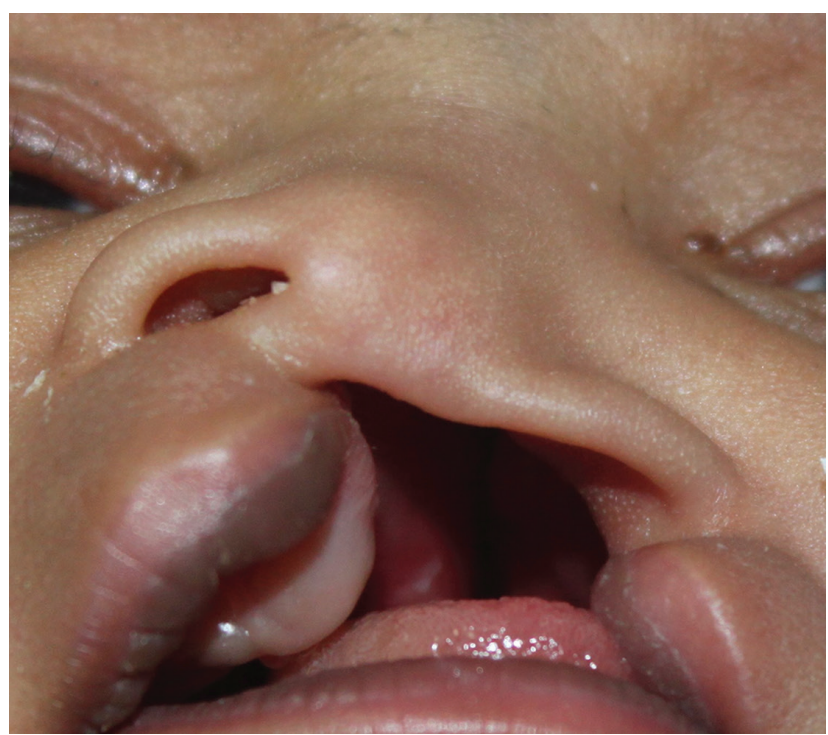

Fig. 1 NAM-basal photograph pre-NAM. Abbreviation: NAM, nasoalveolar molding.

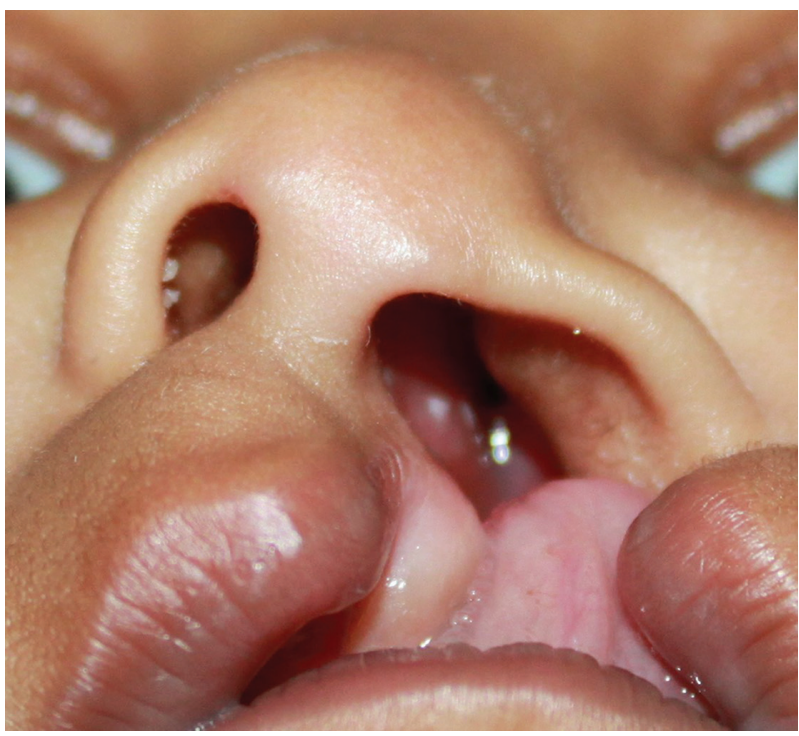

Fig. 2 NAM-basal photograph post-NAM. Abbreviation: NAM, nasoalveolar molding. at 1-year follow-up (T2), at 3-year follow-up (T3), and 5-year follow-up (T4) appointments (-Fig. 3-6). No rhinoplasty had been conducted on any of the patients during the follow-up period.

A ratio of 1:1 basal photographs were used for various measurements, as mentioned in literature. ${ }^{12,16,17}$ A horizontal reference line was constructed by connecting the most inward points at the outer lateral borders of the cleft and noncleft nostrils. ${ }^{17}$ Four linear measurements (nostril height, nostril width, alar base width, and columella height) and one angular measurement (columella deviation angle) were measured directly on the photographs (-Fig. 7-11). To minimize errors in this technique, the linear measurements were evaluated as ratios. All measurements were done by a single independent surgeon who had not treated any of the patients in this study.

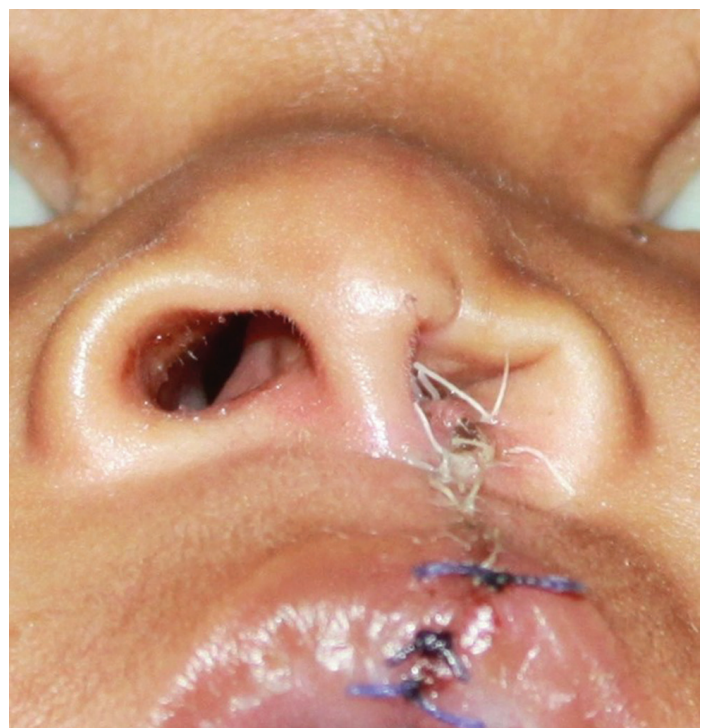

Fig. 3 NAM-basal photograph postcheiloplasty ( $T 1$ follow-up). Abbreviation: NAM, nasoalveolar molding.

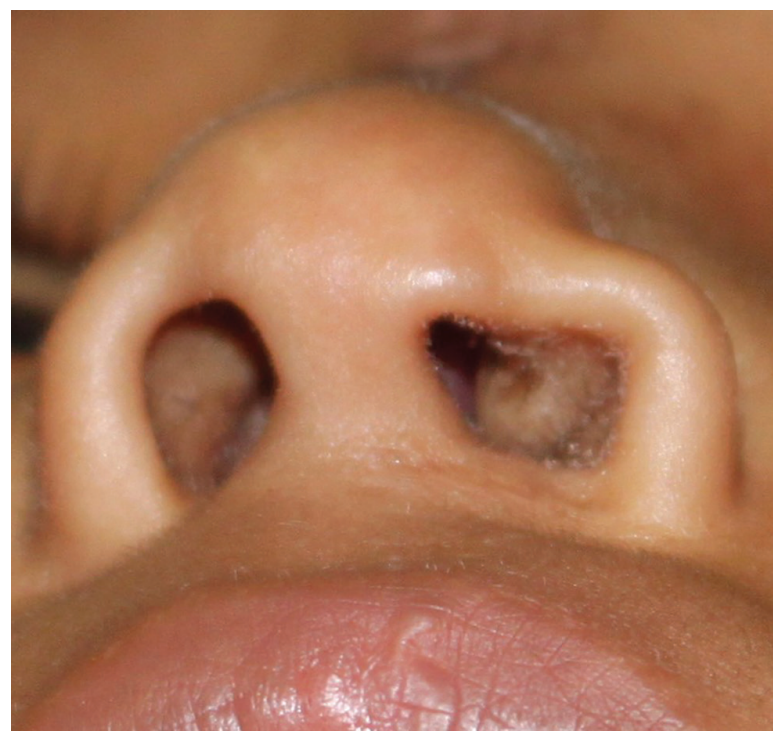

Fig. 4 NAM-basal photograph at 1-year follow-up (T2 follow-up). Abbreviation: NAM, nasoalveolar molding. 


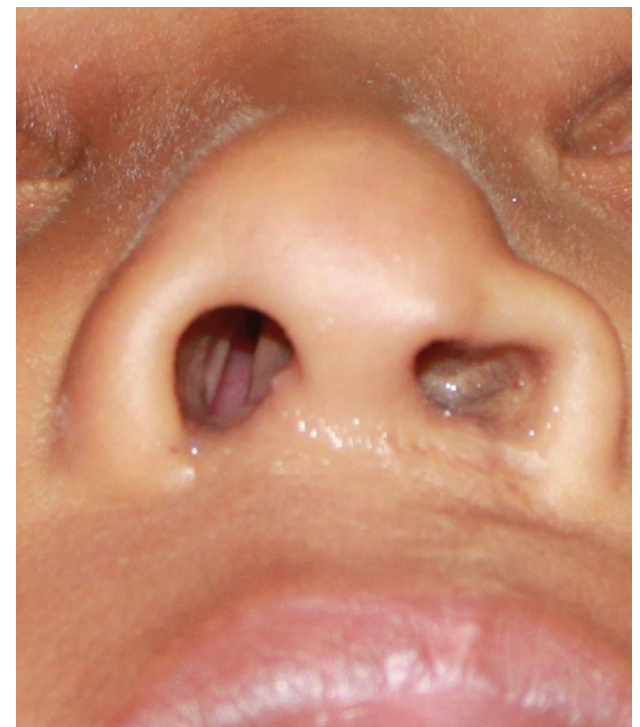

Fig. 5 NAM-basal photograph at 3-year follow-up (T3 follow-up). Abbreviation: NAM, nasoalveolar molding.

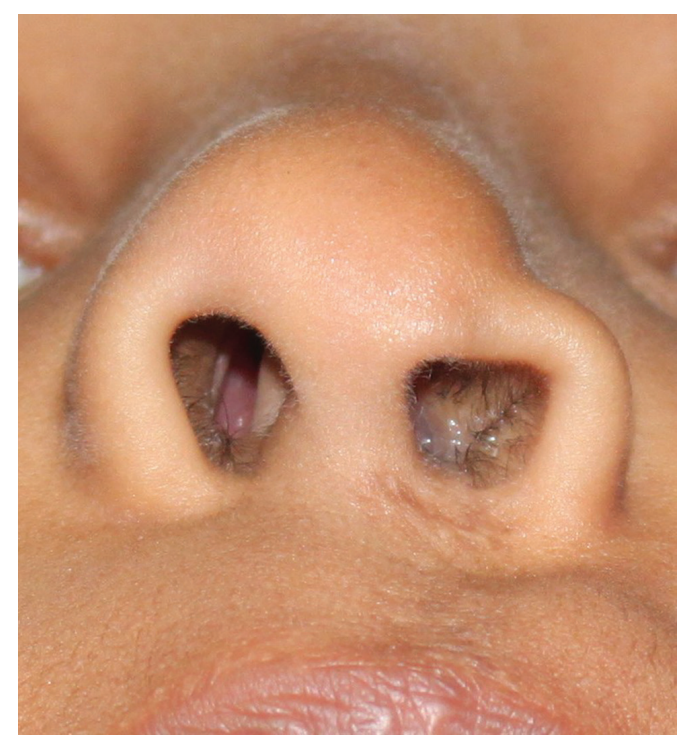

Fig. 6 NAM-basal photograph at 5-year follow-up (T4 follow-up). Abbreviation: NAM, nasoalveolar molding.

Data collected from the study group was analyzed with SPSS version 18 and repeated measures of ANOVA were used to compare the nasal symmetry at each of the intervals to the baseline values. The level of significance was set at $p<0.05$.

\section{Results}

In this retrospective study, the basal photographs of 24 consecutive NAM-treated UCLP patients were studied longitudinally to evaluate the stability of NAM. The nostril height ratio, nostril width ratio, hemicolumella height ratio, alar base width ratio, and columella deviation angle were measured in each of the follow-up photographs.

In the immediate postcheiloplasty period (T1), the nasal height ratio, the nasal width ratio, hemicolumella height and

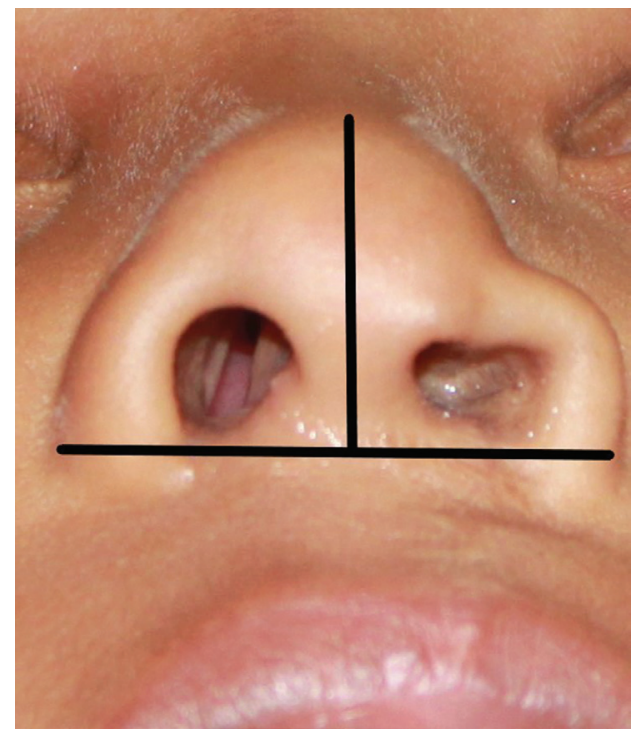

Fig. 7 Columella deviation angle measurement.

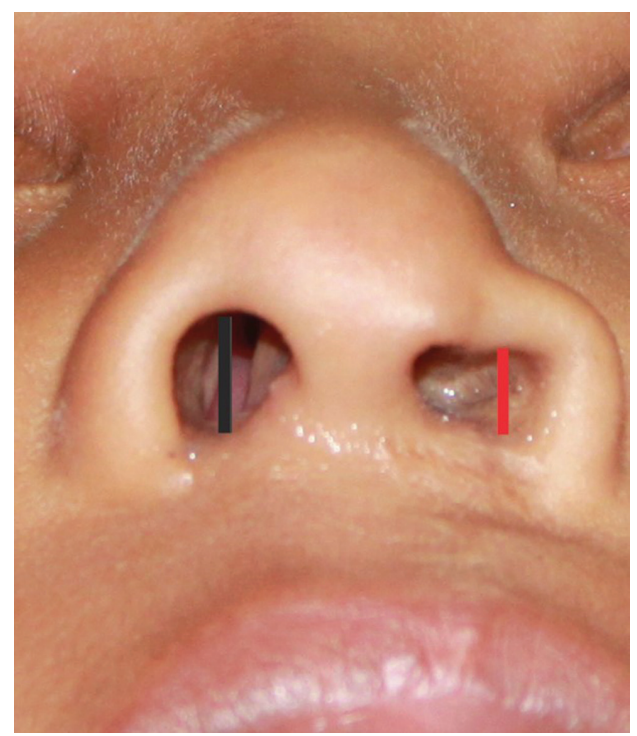

Fig. 8 Nasal height measurement (red-cleft side, black-noncleft side).

alar base width ratio were almost perfectly symmetrical, and the mean columella deviation angle was $89.97^{\circ}$ (- Table 1). In comparison to the baseline values obtained postoperatively (T1), the loss of symmetry was significant across all parameters at all the time durations (T2, T3, and T4) (- Table 3). The mean columella deviation angle was lost by $1.58^{\circ}$ at the end of T2 follow-up $\left(p-0.009^{*}\right), 2.81 \%$ at the T3 follow-up $\left(p-0.002^{*}\right)$ and $3.46 \%$ at T4 follow-up $\left(p<0.001^{*}\right)$. The nostril height on the cleft side was lost by $15.03 \%$ at the T2 follow-up $\left(p<0.001^{*}\right), 18.90 \%$ at T3 follow-up $\left(p<0.001^{*}\right)$, and $22.83 \%$ at the T4 follow-up $\left(p<0.001^{*}\right)$. The nostril width on the cleft side increased by $37.1 \%$ at the T2 follow-up $\left(p<0.001^{*}\right)$, $40.42 \%$ at the T3 follow-up $\left(p<0.001^{*}\right)$, and $40.25 \%$ at the T4 follow-up $\left(p<0.001^{*}\right)$. The hemicolumella length on the cleft side was lost by $13.48 \%$ at the T2 follow-up $\left(p<0.001^{*}\right)$, $19.36 \%$ at the T3 follow-up $\left(p<0.001^{*}\right)$, and $24.89 \%$ at the T4 
follow-up $\left(p<0.001^{*}\right)$. The alar base width on the cleft side increased by $18.89 \%$ at the T2 follow-up $\left(p<0.001^{*}\right), 26.14 \%$ at the T3 follow-up $\left(p<0.001^{*}\right)$, and $40.69 \%$ at the T4 follow-up $\left(p<0.001^{*}\right)$.

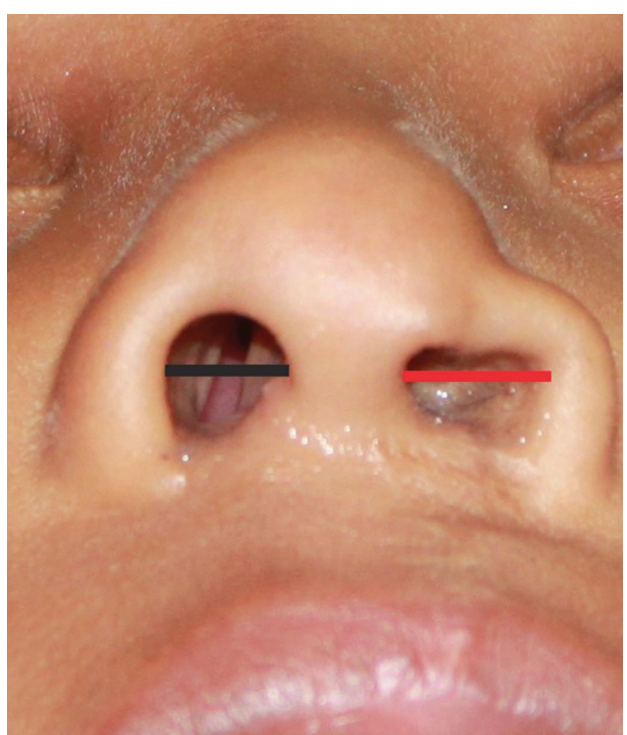

Fig. 9 Nasal width measurement (red-cleft side, black-noncleft side).

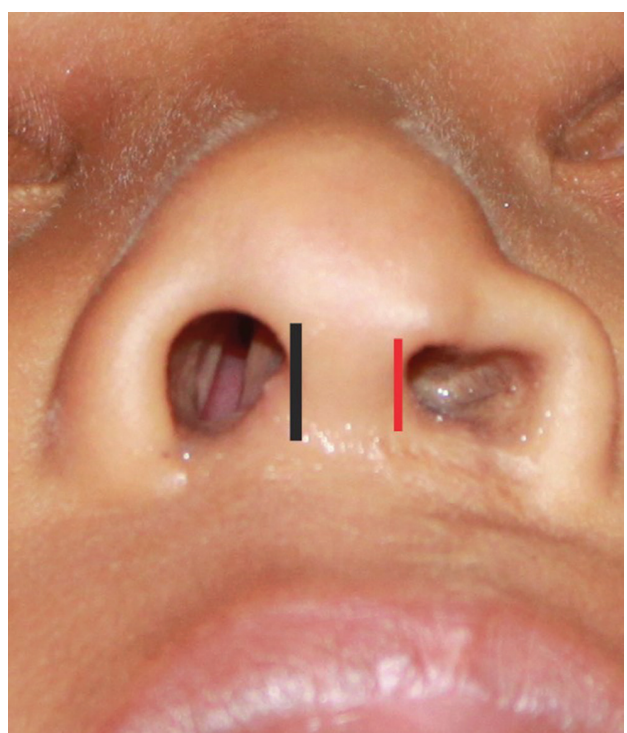

Fig. 10 Columella length measurement (red-cleft side, black-noncleft side).

\section{Discussion}

Grayson's technique of NAM gained popularity as it provided the prospect of reducing the anatomic difficulty and improving the nasal symmetry in early infancy. Although short-term studies have shown positive clinical effects, ${ }^{8-12}$ the technique is relatively new, and fewer studies have documented the stability of the technique in long-term studies. In this retrospective study, we evaluated the stability of the nasal symmetry obtained by NAM at sequential intervals on 2D photographs. Although 2D photographs are dependent on patient position, and may be distorted by parallax effect, they are easy to obtain, inexpensive and noninvasive. Standardized photographic technique and ratios were obtained to minimize errors.

In this study, we found a gradual loss of the mean nasal height, mean gain in nasal width and alar base width, and an increase in the columella deviation from the T1 to the T4 follow-up. Our T2 results were similar to the 1-year results reported by Pai et al, ${ }^{16}$ the 9 -month results reported by Tang et al, ${ }^{1}$ and the 1 -year results reported by Liou et al. ${ }^{17}$ Although the methodology of these studies differed, a loss of symmetry was recorded irrespective of whether only NAM was used-as in our study, a combination NAM and rhinoplasty, ${ }^{17}$ NAM and postoperative nasal stents, ${ }^{17}$ or NAM with rhinoplasty and postoperative nasal stents was used. ${ }^{1}$

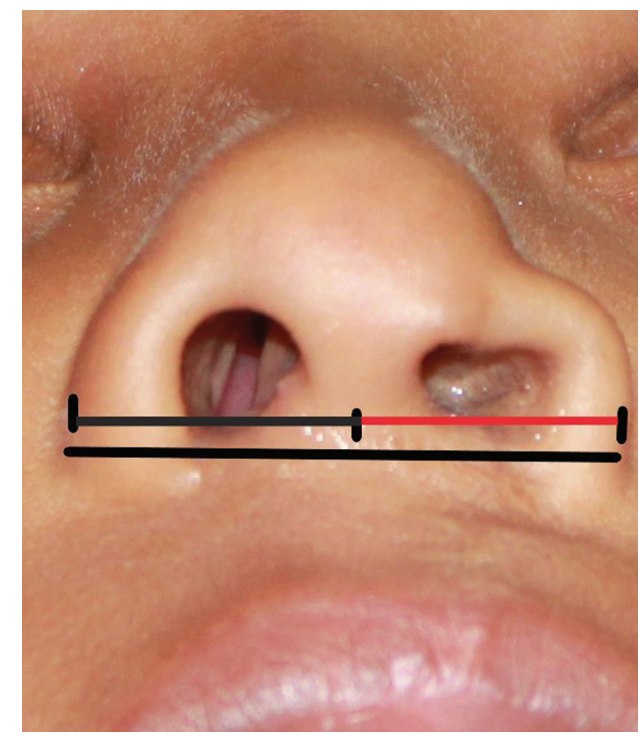

Fig. 11 Alar base width measurement (red-cleft side, blacknoncleft side).

Table 1 Angle and ratios of the various parameters measured

\begin{tabular}{|l|l|l|l|l|}
\hline Parameter & Postcheiloplasty & 1-year follow-up & 3-year follow-up & 5-year follow-up \\
\hline Columella deviation angle & $89.75^{\circ}$ & $88.31^{\circ}$ & $87.21^{\circ}$ & $86.60^{\circ}$ \\
\hline Nasal height ratio & 1.01 & 0.84 & 0.80 & 0.76 \\
\hline Nasal width ratio & 1.04 & 1.38 & 1.41 & 1.41 \\
\hline Columella length ratio & 1.07 & 0.92 & 0.85 & 0.79 \\
\hline Alar base width ratio & 0.97 & 1.13 & 1.21 & 1.34 \\
\hline
\end{tabular}


Table 2 The \% of change in the various parameters from the baseline

\begin{tabular}{|l|l|l|l|l|}
\hline Parameters & \% variation & $\mathbf{1}$ year & $\mathbf{3}$ years & $\mathbf{5}$ years \\
\hline Columella deviation angle & $\mathrm{\%}^{-}$ & $1.58 \%$ & $2.81 \%$ & $3.46 \%$ \\
\hline Nasal height ratio & $\mathrm{\%}^{-}$ & $15.03 \%$ & $18.90 \%$ & $22.83 \%$ \\
\hline Nasal width ratio & $\%$ & $37.10 \%$ & $40.42 \%$ & $40.25 \%$ \\
\hline Columella length ratio & $\%^{-}$ & $13.48 \%$ & $19.36 \%$ & $24.89 \%$ \\
\hline Alar base width ratio & $\%$ & $18.89 \%$ & $26.14 \%$ & $40.69 \%$ \\
\hline
\end{tabular}

Postcheiloplasty follow-up (T1), 1-year follow-up (T2), 3-year follow-up (T3), and 5-year follow-up (T4).

Although the study by Liou et $\mathrm{al}^{17}$ reported a statistically significant loss of symmetry at the 1 st year follow-up, with a $10 \%$ loss of the nostril height, a $20 \%$ gain of the nostril width and a $4.7^{\circ}$ deviation of the columella angle, in contrast to our study, they found a subsequent stability of the nasal symmetry at the 2nd and 3rd year follow-up appointments. This result may be due to the fact that unlike our patients, the NAM treated patients in Liou's study ${ }^{17}$ had received primary rhinoplasty, which may have maintained the results for a longer duration.

The 5-year follow up study reported by Chang et $\mathrm{al}^{2}$ consisted of 76 UCLP patients divided into four groups based on the treatment provided. The first group treated with closed rhinoplasty and postoperative nasal stents. The second group treated with NAM and postoperative nasal stents. The third group with NAM, closed rhinoplasty and postoperative nasal stents. The fourth group with NAM, semiopen rhinoplasty with Tajima's incision, a 20\% surgical overcorrection, and postoperative nasal stents. Our results were similar to their second group which had received NAM and postoperative nasal stents. The nostril height was 0.76 in both the current study and in their study, and nostril width ratio was 1.41 in the current study and 1.36 in their study. Interestingly, although the second group showed a statistically significant improvement in comparison to the first group, there was no statistically significant difference between the second and third groups. Finally, their study concluded that the best symmetry was observed in the patients who had received NAM, semiopen rhinoplasty with $20 \%$ overcorrection and postoperative stents.

Although the methodology used, population studied, and surgeon factors differed in all the previous studies, there seems to be an indication that the differential growth between the cleft and noncleft sides, rather than the initial technique, is responsible for the loss of symmetry. ${ }^{17}$ Although primary rhinoplasty appears to stabilize the results in the early follow-up duration, ${ }^{17}$ the continuing growth may probably override the initial symmetry gained. The $20 \%$ overcorrection suggested by Chang et $\mathrm{el}^{2}$ probably equals the lag on the cleft side due to which the results were symmetrical in their study.

\section{Limitations}

This study was retrospective and done on 2D photographs. Although the conventional photography offers the benefit of low-cost and quick data collection, it is limited by patient head position and the parallax effect. The photographs were also studied in the basal view which is generally not viewed in social circumstances. The method of measurement has not been validated. The study also stops at 5 years of age and includes only 24 patients.

\section{Conclusion}

In this study, we found that NAM was a useful adjuvant in achieving nasal symmetry in patients with UCLP in the immediate postoperative period. However, as the patients aged, there was a gradual loss of symmetry. Although there is no conclusive evidence, the loss of symmetry may be due to the unequal growth on the cleft and noncleft sides. These patients will be followed-up till end of growth for a definite conclusion on the long-term effect of NAM.

\section{Conflict of interest}

We have no conflict of interest to declare.

\section{References}

1 Tang PM, Chao NS, Leung MW, Kelvin KW. Changes in nasal configuration following primary rhinoplasty: Direct anthropometric measurement in patients with complete unilateral cleft lip and palate. Cleft Palate Craniofac J 2016;53(5):557-561

2 Chang CS, Por YC, Liou EJ, Chang CJ, Chen PK, Noordhoff MS. Long-term comparison of four techniques for obtaining nasal symmetry in unilateral complete cleft lip patients: a single surgeon's experience. Plast Reconstr Surg 2010;126(4):1276-1284

3 Hosseinian B, Rubin MS, Clouston SAP, et al. Comparative analysis of three-dimensional nasal shape of casts from patients with unilateral cleft lip and palate treated at two institutions following rotation advancement only (Iowa) or nasoalveolar molding and rotation advancement in conjunction with primary rhinoplasty (New York) Cleft Palate Craniofac J 2018;55(10):1350-1357

4 Salyer KE, Genecov ER, Genecov DG. Unilateral cleft lip-nose repair: a 33-year experience. J Craniofac Surg 2003;14(4): 549-558

5 Lu TC, See UC, Chen PKT, Noordhoff MS. Unilateral Complete Cleft Lip Repair with Primary Semi-open Rhinoplasty. In: Anh Tran T, Panthaki Z, Hoballah J, Thaller S, eds. Operative Dictations in Plastic and Reconstructive Surgery. Springer, Cham; 2017

6 Grayson BH, Santiago PE, Brecht LE, Cutting CB. Presurgical nasoalveolar molding in infants with cleft lip and palate. Cleft Palate Craniofac J 1999;36(6):486-498

7 Grayson BH, Shetye PR. Presurgical nasoalveolar moulding treatment in cleft lip and palate patients. Indian J Plast Surg 2009;42(1(Suppl):S56-S61

8 Lee CT, Garfinkle JS, Warren SM, Brecht LE, Cutting CB, Grayson BH. Nasoalveolar molding improves appearance of 
children with bilateral cleft lip-cleft palate. Plast Reconstr Surg 2008;122(4):1131-1137

9 Liou EJ, Subramanian M, Chen PK. Progressive changes of columella length and nasal growth after nasoalveolar molding in bilateral cleft patients: a 3-year follow-up study. Plast Reconstr Surg 2007;119(2):642-648

10 Meazzini MC, Rossetti G, Morabito A, Garattini G, Brusati R. Photometric evaluation of bilateral cleft lip and palate patients after primary columella lengthening. Cleft Palate Craniofac J 2010;47(1):58-65

11 Nazarian Mobin SS, Karatsonyi A, Vidar EN, et al. Is presurgical nasoalveolar molding therapy more effective in unilateral or bilateral cleft lip-cleft palate patients? Plast Reconstr Surg 2011;127(3):1263-1269

12 Zuhaib M, Bonanthaya K, Parmar R, Shetty PN, Sharma P. Presurgical nasoalveolar moulding in unilateral cleft lip and palate. Indian J Plast Surg 2016;49(1):42-52
13 Chen PK-T, Noordhoff MS, Liou EJW. Treatment of Complete Bilateral Cleft Lip-Nasal Deformity. Semin Plast Surg 2005;19: 329-342

14 Matsuo K, Hirose T. Nonsurgical correction of cleft lip nasal deformity in the early neonate. Ann Acad Med Singapore 1988;17(3):358-365

15 Sommerlad BC. A technique for cleft palate repair. Plast Reconstr Surg 2003;112(6):1542-1548

16 Pai BC, Ko EW, Huang CS, Liou EJ. Symmetry of the nose after presurgical nasoalveolar molding in infants with unilateral cleft lip and palate: a preliminary study. Cleft Palate Craniofac J 2005;42(6):658-663

17 Liou EJ, Subramanian M, Chen PK, Huang CS. The progressive changes of nasal symmetry and growth after nasoalveolar molding: a three-year follow-up study. Plast Reconstr Surg 2004;114(4):858-864 